\title{
Analysis of Trends in Cancer-related Mortality in Turkey
}

\author{
(D) Nurhan DOĞAN,' 1 Turgut KAÇAN, ${ }^{2}$ (D) İsmet DOĞAN' \\ 'Department of Biostatistics and Medical Informatics, Afyonkarahisar University of Health Sciences, Afyonkarahisar-Turkey \\ ${ }^{2}$ Department of Medical Oncology, Bursa High Specialized Training and Research Hospital, Bursa-Turkey
}

\begin{abstract}
OBJECTIVE
Cancer is one of the most important causes of death, both in Turkey and in the world. Cancer also causes economic burden to countries. Therefore, it is important to evaluate the data and mortality trends related to cancer using statistical methods to take primary or secondary preventive measures. In this study, we aimed to analyse the recent changes in cancer mortality via using Joinpoint Regression Analysis (JRA) method between 2009 and 2017.
\end{abstract}

\section{METHODS}

The data on cancer-related mortality in Turkey from the database of the Turkish Statistical Institute (TurkStat) were obtained and analysed with the JRA method. Age-standardized mortality rate (ASR), annual percent change (APC) and average annual percent change (AAPC) were calculated and compared according to gender.

\section{RESULTS}

In female patients, the significant increase in mortality trends were observed in breast, gastrointestinal system (liver, pancreas, and colon) and gynecological cancers (ovarian and corpus uteri). In male patients, liver, pancreas, colon, lip, oral cavity, pharynx and bladder cancers were shown to increase significantly. There was no significant increase in other cancer sites.

\section{CONCLUSION}

To our knowledge, this study is the first study that has analyzed cancer and cancer-related death trends using JRA at the national level in Turkey. We think that our study can demonstrate the contrast trends in cancer rates. According to these results, better health interventions could be developed to fight against cancer and to reduce the incidence of cancer-related morbidity and mortality.

Keywords: Cancer; joinpoint regression analysis; mortality; Turkey.

Copyright $\odot$ 2020, Turkish Society for Radiation Oncology

\section{Introduction}

Cancer is one of the most important public health problem and cause of death both in Turkey and in the world. The number of cases and deaths is expected to increase rapidly due to growth and aging of the population, changes in lifestyle behaviours, including tobacco use, sedentary lifestyle, high body mass index (BMI), and reproductive patterns that increase cancer risk, especially in developing countries.[1]

The increasing number of deaths due to cancer is responsible for its high mortality worldwide. In 2012, there were 8.2 million cancer-related deaths all over the world. The most common causes of cancer-related 
deaths were respiratory and gastrointestinal system cancers.[2] Thanks to early diagnosis and treatment of cancer, cancer morbidity and mortality have started to decrease in developed countries; however, they still continue to increase in developing countries.[1] In 2018, the mortality rate was estimated to be $57.3 \%$ in Asia, $20.3 \%$ in Europe and $14.4 \%$ in America.[3] The leading cause of cancer-related death is lung cancer in men $(22.0 \%)$ and breast cancer (15.0\%) in women. Thus, it is important to detect the most important causes of cancer-related death and to take preventive measures against.[3]

In modelling trends over time, many statistical methods have been developed to detect the changes in cancer trends and deaths. One of them is the joinpoint regression method that is used to detect changes and determine the trends between joinpoints. Cancer not only causes morbidity and mortality in patients with cancer but also causes economic burden on countries. Therefore, planning prevention programs against cancer and evaluating their efficiency by monitorization of changing trends on cancer statistics, especially mortality, are highly important for countries. Herein, our goal is to reveal alterations in the cancer-related death rates in Turkey (2009-2017).

\section{Materials and Methods}

\section{Data Collection}

Mortality data of cancer in Turkey between the years 2009 to 2017 were accessed over the Turkish Statistical Institute death database.[4] WHO ICD code (revised version 10) was the reference on this data collection. [5] Different cancer sites and their ICD-10 codes are listed here: lip, oral cavity, pharynx (C00-C14), oesophagus (C15), stomach (C16), colon (C18), rectum and anus (C19-C21), liver (C22), pancreas (C25), larynx, lung and bronchus (C32-C34), skin (C43), breast (C50), cervix uteri (C53), corpus uteri and part unspecified (C54-C55), ovary (C56), prostate (C61), kidney (C64), bladder (C67), lymphoid, hematopoietic and related tissue (C81-C96).[5] Analyses were stratified according to the male and female gender. Age-standardized death rates per 100.000 people (WHO Standard population) were determined in order to make direct standardization. [6] Ages were categorized by 5-year age groups for standardization purposes, which is also beneficial to make comparisons with other countries.

The number of deaths for each cancer site was presented according to gender and age [year] (age categories: 0-4, 5-9, 10-14, 15-19, 20-24, 25-29, 30-34, 35-
$39,40-44,45-49,50-54,55-59,60-64,65-69,70-74$ and $\geq 75$ years).

\section{Statistical Analysis}

JRA is a statistical modelling approach, which is generally used for modelling the time trends in a data set. JRA begins with the minimum number of joinpoints and tests whether one or more joinpoints are statistically significant and should be added to the model. The number of joinpoints is determined by Monte Carlo permutation tests.[7] The final model shows the best fitting joinpoints, where the rate changes significantly. Each joinpoint provides information about statistically significant changes, estimated APC and AAPC that are computed with their $95 \%$ confidence intervals (95\%CI). AAPC is the geometric mean of the annual changes from all of the partitions.[8,9] Analyses were carried out using Joinpoint Regression Program software version 4.6.0.0-2018 provided by the surveillance research program of the National Cancer Institute, USA.[10] The AAPC for death rates were calculated with the $95 \%$ confidence interval.

The first step is applying the simplest model to the data (e.g. for cancer rates) on a log scale using this formula: $\ln$ (age-adjusted death rate) $=a+b x$, where $x$ represents the age of death. APC is computed as $\left[100^{*}(\mathrm{eb}\right.$ -1)]. APC is statistically significant if its confidence interval does not contain the zero value. Since our study included nine years, we allowed only two points as a maximum number for analysis.[11] The significance level for the analyses was 0.05 .

\section{Results}

The number of deaths and ASRs according to major cancer sites in men and women in the period of 20092017 are presented in Table 1. In 2017, the three leading cancer sites that caused death in male and their ASRs were estimated to be lung (ASR, 47.5), stomach (ASR, 9.9), lymphoid and hematopoietic (ASR, 8.4). Lung cancer was the main cause of cancer death. For the same year, breast cancer (ASR, 8.0) was projected to be the leading cause of cancer-related death in female, followed by larynx, lung and bronchus (ASR, 6.9) and lymphoid and hematopoietic (ASR, 5.3). ASR change in percentage was varying according to the site of cancer. ASRs for colon, liver and pancreatic cancer were over $20 \%$ in both male and female. In male, although the lip, oral cavity, pharynx cancers revealed the highest increase in ASRs by $85 \%$, the lowest decrease was revealed in rectum and anus cancer by $6 \%$ for men. In female, the highest 
Table 1 The number of deaths and age-standardized death rates for major cancers in Turkey, male and female, 2009-2017

\begin{tabular}{|c|c|c|c|c|c|c|c|c|c|c|}
\hline & \multicolumn{4}{|c|}{2009} & \multicolumn{4}{|c|}{2017} & & \\
\hline & \multicolumn{2}{|c|}{ Male } & \multicolumn{2}{|c|}{ Female } & \multicolumn{2}{|c|}{ Male } & \multicolumn{2}{|c|}{ Female } & \multicolumn{2}{|c|}{ ASR\% Change } \\
\hline & Death (n) & ASR & Death (n) & ASR & Death (n) & ASR & Death (n) & ASR & Male & Female \\
\hline Lip, oral cavity, pharynx & 429 & 1.3 & 202 & 0.5 & 578 & 2.4 & 306 & 0.6 & 85 & 20 \\
\hline Esophagus & 351 & 1.0 & 268 & 0.7 & 502 & 1.1 & 338 & 0.6 & 10 & -14 \\
\hline Stomach & 3216 & 9.5 & 1790 & 4.3 & 4465 & 9.9 & 2222 & 4.1 & 4 & -5 \\
\hline Colon & 2019 & 5.9 & 1619 & 3.8 & 3485 & 7.7 & 2606 & 4.7 & 31 & 24 \\
\hline Rectum and anus & 610 & 1.8 & 396 & 1.0 & 769 & 1.7 & 520 & 0.9 & -6 & -10 \\
\hline Liver & 1290 & 3.8 & 693 & 1.7 & 2094 & 4.7 & 1174 & 2.1 & 24 & 24 \\
\hline Pancreas & 1737 & 5.2 & 1169 & 2.9 & 2824 & 6.3 & 2094 & 3.8 & 21 & 31 \\
\hline Larynx, lung and bronchus & 15625 & 47.3 & 2582 & 6.4 & 21017 & 47.5 & 3731 & 6.9 & 0 & 8 \\
\hline Skin & 196 & 0.6 & 154 & 0.4 & 268 & 0.6 & 214 & 0.4 & 0 & 0 \\
\hline Breast & 68 & 0.2 & 2550 & 6.5 & 72 & 0.2 & 4443 & 8.0 & 0 & 23 \\
\hline Cervix uteri & - & - & 406 & 1.1 & - & - & 476 & 0.9 & - & -18 \\
\hline Corpus uteri and part unspecified & - & - & 582 & 1.5 & - & - & 964 & 1.8 & - & 20 \\
\hline Ovary & - & - & 993 & 2.6 & - & - & 1547 & 3.1 & - & 19 \\
\hline Prostate & 2652 & 7.5 & - & - & 3687 & 7.7 & - & - & 3 & - \\
\hline Kidney & 504 & 1.5 & 263 & 0.7 & 757 & 1.7 & 337 & 0.6 & 13 & -14 \\
\hline Bladder & 1168 & 3.5 & 224 & 0.5 & 1863 & 4.1 & 396 & 0.6 & 17 & 20 \\
\hline Lymphoid and hematopoietic & 2943 & 8.5 & 2062 & 5.2 & 3712 & 8.4 & 2704 & 5.3 & -1 & 2 \\
\hline
\end{tabular}

ASR: Age-Standardized rates

increase in ASRs was observed for pancreas cancer by $31 \%$ and the lowest decrease in ASRs was observed for at cervix uteri by $18 \%$. The results of the JRA, APC and AAPC for both genders are shown in Table 2. Evaluated according to the cancer sites, a single joinpoint model is found to be the best-fit model for male deaths rates in the lip-oral cavity-pharynx, colon and larynx-lungbronchus cancers. ASRs from lip-oral cavity-pharynx cancer decreased from the year 2009 till 2015 (APC: -0.2). After 2015, ASRs increased significantly (APC: 31.8). ASRs from colon cancer increased (APC: 5.4) from 2009 to 2014. And after 2014, ASRs decreased significantly (APC: -0.2). Similarly, ASRs from larynx, lung and bronchus cancers also displayed a rising trend from 2009 to 2013, followed by a significant decrease except between 2013-2017 (APC: -2.2). For other cancer sites, the zero joinpoint model was determined as the best model. A steady, significant increase was observed in only three cancer sites (liver, pancreas, bladder) during the analysed period.

One joinpoint model (in 2003) was obtained as the best-fit model for female deaths rates in the stomach and larynx-lung-bronchus cancers. ASRs from stomach cancer were shown to increase by $1.8 \%$ per year from 2009 to 2014 , and to decrease significantly by
4.5\% per year after 2014. ASRs from larynx, lung and bronchus cancers also were shown to have a significant increase trend from 2009 to 2015 , followed by a decline between the years 2015-2017 (APC: -2.4).

In other cancer sites, the zero joinpoint model was determined as the best model. A steady, significant increase was observed in six cancer sites: colon, liver, pancreas, breast, corpus uteri and unspecified, ovary during the analysis period (2009-2017).

\section{Discussion}

Cancer with cardiovascular diseases is the main etiological factors for morbidity and death globally. Cancer is the first or second leading cause of death in nearly 100 countries. [3] It is also publicly announced that approximately 18 million new cases and 10 million cancer deaths were estimated to happen in 2018 worldwide. [12] The risk of cancer varies according to genetic risk factors, regional variations, lifestyle and environmental risk factors that some of which can be changeable. $[13,14]$ Thus, it is important to take precautions. Many countries adopted preventive measures to reduce the cancer burden at the World Health Assembly and have built a global action plan.[12] 
Table 2 Trends in age-standardized death rates for cancers in Turkey according to joinpoint analysis, 2009-2017 (Male, Female)

\begin{tabular}{|c|c|c|c|c|c|}
\hline & \multirow{2}{*}{$\begin{array}{c}\text { AAPC }(95 \% \mathrm{Cl}) \\
\text { Full Range }\end{array}$} & \multicolumn{2}{|c|}{ Trend 1} & \multicolumn{2}{|c|}{ Trend 2} \\
\hline & & Period & $\operatorname{APC}(95 \% \mathrm{CI})$ & Period & APC $(95 \% \mathrm{CI})$ \\
\hline \multicolumn{6}{|l|}{ Males } \\
\hline Lip, oral cavity, pharynx & $7.0^{\wedge}(0.3-11.2)$ & 2009-2015 & $-0.2(-3.4 ; 3.2)$ & 2015-2017 & $31.8 \wedge(8.6 ; 59.9)$ \\
\hline Esophagus & $-0.2(-3.6 ; 3.3)$ & 2009-2017 & $-0.2(-3.6 ; 3.3)$ & & \\
\hline Stomach & $0.5(-1.1 ; 2.2)$ & 2009-2017 & $0.5(-1.1 ; 2.2)$ & & \\
\hline Colon & $3.2 \wedge(1.4 ; 5.1)$ & 2009-2014 & $5.4 \wedge(2.8 ; 8.0)$ & 2014-2017 & $-0.2(-5.5 ; 5.4)$ \\
\hline Rectum and anus & $-0.4(-1.8 ; 1.1)$ & 2009-2017 & $-0.4(-1.8 ; 1.1)$ & & \\
\hline Liver & $2.1 \wedge(1.0 ; 3.2)$ & $2009-2017$ & $2.1^{\wedge}(1.0 ; 3.2)$ & & \\
\hline Pancreas & $2.6 \wedge(1.1 ; 4.0)$ & 2009-2017 & $2.6 \wedge(1.1 ; 4.0)$ & & \\
\hline Larynx, lung and bronchus & $0.1(-0.4 ; 0.6)$ & $2009-2013$ & $2.4 \wedge(1.5 ; 3.4)$ & 2013-2017 & $-2.2^{\wedge}(-3.1 ;-1.2)$ \\
\hline Skin & $2.3(-2.0 ; 6.7)$ & $2009-2017$ & $2.3(-2.0 ; 6.7)$ & & \\
\hline Breast & $-1.6(-9.8 ; 7.3)$ & $2009-2017$ & $-1.6(-9.8 ; 7.3)$ & & \\
\hline Cervix uteri & - & & & & \\
\hline Corpus uteri and part unspecified & - & & & & \\
\hline Ovary & - & & & & \\
\hline Prostate & $0.3(-1.2 ; 1.9)$ & $2009-2017$ & $0.3(-1.2 ; 1.9)$ & & \\
\hline Kidney & $1.6(-0.4 ; 3.6)$ & 2009-2017 & $1.6(-0.4 ; 3.6)$ & & \\
\hline Bladder & $2.3 \wedge(0.7 ; 3.9)$ & $2009-2017$ & $2.3 \wedge(0.7 ; 3.9)$ & & \\
\hline Lymphoid and hematopoietic & $0.3(-1.0 ; 1.6)$ & 2009-2017 & $0.3(-1.0 ; 1.6)$ & & \\
\hline \multicolumn{6}{|l|}{ Females } \\
\hline Lip, oral cavity, pharynx & $1.7(-0.3-3.9)$ & 2009-2017 & $1.7(-0.3-3.9)$ & & \\
\hline Esophagus & $-0.1(-2.4 ; 2.3)$ & 2009-2017 & $-0.1(-2.4 ; 2.3)$ & & \\
\hline Stomach & $-0.6(-2.5 ; 1.3)$ & $2009-2014$ & $1.8(-0.8 ; 4.4)$ & 2014-2017 & $-4.5(-9.7 ; 1.1)$ \\
\hline Colon & $2.1 \wedge(1.2 ; 3.0)$ & 2009-2017 & $2.1^{\wedge}(1.2 ; 3.0)$ & & \\
\hline Rectum and anus & $-1.2(-3.4 ; 1.0)$ & 2009-2017 & $-1.2(-3.4 ; 1.0)$ & & \\
\hline Liver & $3.1 \wedge(1.8 ; 4.4)$ & $2009-2017$ & $3.1^{\wedge}(1.8 ; 4.4)$ & & \\
\hline Pancreas & $3.4 \wedge(1.9 ; 4.9)$ & $2009-2017$ & $3.4 \wedge(1.9 ; 4.9)$ & & \\
\hline Larynx, lung and bronchus & $1.2(-0.5 ; 3.0)$ & $2009-2015$ & $2.5^{\wedge}(1.0 ; 6.4)$ & 2015-2017 & $-2.4(-10.6 ; 6.4)$ \\
\hline Skin & $0.8(-2.0 ; 3.6)$ & $2009-2017$ & $0.8(-2.0 ; 3.6)$ & & \\
\hline Breast & $2.7 \wedge(1.0 ; 4.5)$ & $2009-2017$ & $2.7 \wedge(1.0 ; 4.5)$ & & \\
\hline Cervix uteri & $-0.1(-3.0 ; 1.1)$ & $2009-2017$ & $-0.1(-3.0 ; 1.1)$ & & \\
\hline Corpus uteri and part unspecified & $2.9 \wedge(1.3 ; 4.6)$ & $2009-2017$ & $2.9 \wedge(1.3 ; 4.6)$ & & \\
\hline Ovary & $1.8^{\wedge}(0.2 ; 3.5)$ & $2009-2017$ & $1.8^{\wedge}(0.2 ; 3.5)$ & & \\
\hline Prostate & - & - & - & & \\
\hline Kidney & $-0.3(-2.3 ; 1.8)$ & $2009-2017$ & $-0.3(-2.3 ; 1.8)$ & & \\
\hline Bladder & $2.0(-0.3 ; 4.4)$ & $2009-2017$ & $2.0(-0.3 ; 4.4)$ & & \\
\hline Lymphoid and hematopoietic & $0.3(-1.0 ; 1.6)$ & 2009-2017 & $0.3(-1.0 ; 1.6)$ & & \\
\hline
\end{tabular}

AAPC: Average annual percent change; Cl: Confidence interval; APC :Annual percent change; $\wedge$ APC and AAPC are statistically significantly different from zero (two-sided $\mathrm{P}<0.05$ )

In Turkey, the government has also adopted precautions nationally. Turkey bridges between Asia and Europe geographically. As mentioned before, the risk of cancer varies according to genetic risks, regional variations, lifestyle and environmental risk factors. Therefore, cancer types vary in the West and East Regions of Turkey.[15]

To our knowledge, this study is the first epidemiological study analysing the cancer trends in Turkey using the JRA method. This study also has the importance of being the most actual analysis investigating the cancer deaths within the country. The used analysis in this study, namely the JRA is usually applied to analyse varying trends over time. In addition to providing important baseline data on present cancer deaths, this study also demonstrates contrasting trends in cancer rates over the past nine years. Our study is an answer to the question of whether cancer 
mortality trends are increasing or decreasing over the years in Turkey.

According to our analysis, the three leading causes of cancer-related death in male patients were lung, gastric and haematological cancers. The leading cause of cancer-related mortality on male patients was lung cancer. In females, breast cancer was the leading cause of cancer-related deaths. The next leading causes were respiratory system and haematological cancers. While ARSs of colon, liver and pancreatic cancers were equal (over 20\%), head and neck cancers had the highest increase in males, whereas pancreatic cancers had the highest increase in females.

As shown in Table 2, ASRs of head and neck cancers decreased between 2009 and 2015 among male patients, whereas between 2015 and 2017, ASRs of head and neck cancers increased according to a single joinpoint model. ASRs of colon cancer increased between 2009 and 2014, and after that, a decrease started significantly. Similarly, ASRs of respiratory system cancers showed an upward trend between 2009 and 2013, followed by a significant decline between 20132017. According to these results, a single joinpoint model is found to be the best-fit model for male death rates in these cancers.

For other cancer sites, the zero joinpoint model was determined to be the best model. A steady, significant increase was observed in only three cancer sites (liver, pancreas and bladder) during the nine-year period of 2009-2017.

As known, one joinpoint model was the best model for female death rates in some cancers. ASRs of gastric cancer increased in the first five-year period (2009-14), then, decreased in the second four-year period (201417). ASRs of respiratory system cancers showed a significant upward trend from 2009 to 2015, followed by the decline for the period of 2015-2017.

In other cancer sites, the zero joinpoint model was determined to be the best model. A steady, significant increase was observed in six cancer sites (colon, liver, pancreas, breast, corpus uteri and unspecified, ovary) during the whole analysis period (2009-2017).

\section{Limitations of the Study}

No mortality data were unavailable before 2009 to compare. No comparisons were available according to regional differences, which is important as the percentages of cancers differ according to geographical regions. The other limitation was the short analysis period. In general, change in rates in morbidity and mortality of cancers is a long-lasting process.

\section{Conclusion}

To our knowledge, this study is the first study that has analyzed cancer and cancer-related death trends using JRA at the national level in Turkey. We think that our study is demonstrating the contrast trends in cancer rates. According to these results, better health interventions could be developed to fight against cancer and to reduce the incidence of cancer-related morbidity and mortality.

Peer-review: Externally peer-reviewed.

Conflict of Interest: The authors declare no conflict of interest.

Financial Support: No financial support has used for the study.

Authorship contributions: Concept - N.D.; Design - N.D., T.K., İ.D.; Supervision - N.D., T.K., İ.D.; Funding - None; Materials - None; Data collection and/or processing - N.D., İ.D.; Data analysis and/or interpretation - N.D., T.K., İ.D.; Literature search - N.D., T.K., İ.D.; Writing - N.D., T.K., İ.D.; Critical review - N.D., T.K., İ.D.

\section{References}

1. Torre LA, Siegel RL, Ward EM, Jemal A. Global cancer incidence and mortality rates and trends-an update. Cancer Epidemiology and Prevention Biomarkers 2016;25(1):16-27.

2. Ferlay J, Soerjomataram I, Ervik M, Dikshit R, Eser S, Mathers C, et al. GLOBOCAN 2012 v1.0, Cancer Incidence and Mortality Worldwide: IARC Cancer Base No. 11. Lyon, France: International Agency for Research on Cancer, 2013.

3. Bray F, Ferlay J, Soerjomataram I, Siegel RL, Torre LA, Jemal A. Global cancer statistics 2018: GLOBOCAN estimates of incidence and mortality worldwide for 36 cancers in 185 countries. CA Cancer J Clin 2018;68(6):394-424.

4. TurkStat, Turkish Statistical Institute, Cause of Death Statistics. Available from: www.turkstat.gov.tr.

5. WHO. International statistical classification of disease and related health problems. 10th revision. Volume 2. Geneva; 2008. Available from: https://icd.who. int/browse10/Content/statichtml/ICD10Volume2_ en_2008.pdf.

6. Ahmad OB, Boschi-Pinto C, Lopez AD, Murray CJL, Lozano R, Inoue M. Age Standardization of Rates: a new Who Standard. WHO, 2001. Available from: https://www.who.int/healthinfo/paper31.pdf?ua=1.

7. Kim HJ, Fay MP, Feuer EJ, Midthune DN. Permutation tests for joinpoint regression with applications to cancer rates. Stat Med 2000;19(3):335-51. 
8. Clegg LX, Hankey BF, Tiwari R, Feuer EJ, Edwards BK. Estimating average annual per cent change in trend analysis. Stat Med 2009;28(29):3670-82.

9. Kim, HJ, Luo J, Chen HS, Green D, Buckman D, Byrne J, et al. Improved confidence interval for average annual percent change in trend analysis. Stat Med 2017;36(19):3059-74.

10. National Cancer Institute. Joinpoint Regression Program, Version 4.7.0.0. February, 2019. Available from: https://surveillance.cancer.gov/joinpoint/.

11. National Cancer Institute. Joinpoint Help Manual, Version 4.6.0.0. Surveillance Research Program, April 2018. Available from: https://surveillance.cancer.gov/ joinpoint/Joinpoint_Help_4.6.0.0.pdf.

12. World Health Organization. International Agency for Research on Cancer, Latest global cancer data,
Press release No:263, Geneva, Switzerland, 2018. Available from: https://www.iarc.fr/wp-content/uploads/2018/09/pr263_E.pdf

13. Wilson LF, Antonsson A, Green AC, Jordan SJ, Kendall BJ, Nagle CM, et al. How many cancer cases and deaths are potentially preventable? Estimates for Australia in 2013. Int J Cancer 2018;142(4):691-701.

14. Brown KF, Rumgay H, Dunlop C, Ryan M, Quartly F, Cox A, et al. The fraction of cancer attributable to modifiable risk factors in England, Wales, Scotland, Northern Ireland, and the United Kingdom in 2015. Br J Cancer 2018;118(8):1130-41.

15. Turkiye Kanser İstatistikleri. T.C. Sağlık Bakanlığı, Türkiye Halk Sağlığı Kurumu, Ankara, 2015. Available from: https://docplayer.biz.tr/6567493-Turkiyekanser-istatistikleri.html. 